\title{
Regenerative potential of human nucleus pulposus resident stem/progenitor cells declines with ageing and intervertebral disc degeneration
}

\author{
HAO WU $^{1 *}$, YUPAN SHANG ${ }^{2 *}$, JIAYUE YU $^{2 *}$, XIAOLI ZENG ${ }^{2}$, JINHUA LIN $^{2}$, \\ MEI TU ${ }^{3}$, LEK HANG CHEANG ${ }^{4}$ and JIAQING ZHANG ${ }^{2}$
}

${ }^{1}$ Department of Orthopaedic Surgery, The First Affiliated Hospital, Jinan University, Guangzhou, Guangdong 510630;
${ }^{2}$ Department of Biochemistry and Molecular Biology, School of Preclinical Medicine, Jinan University;
${ }^{3}$ Department of Materials Science and Engineering, Jinan University, Guangzhou, Guangdong 510632;
${ }^{4}$ Orthopaedic Surgery, Centro Hospitalar Conde de Sao Januario, Macao 999078, P.R. China

Received March 20, 2018; Accepted July 4, 2018

DOI: $10.3892 / \mathrm{ijmm} .2018 .3766$

\begin{abstract}
Numerous studies have demonstrated the presence of resident nucleus pulposus stem/progenitor cells (NPSCs) in the tissue of the intervertebral disc (IVD). However, the cellular identity of NPSCs during IVD degeneration and ageing are poorly defined at present, despite significant progress in the understanding of NPSC biology. In the present study, NPSCs were isolated from human degenerated IVD and were characterized by flow cytometry, gene expression assays and proliferation and multipotency analysis. The results of the present study demonstrated that NPSCs isolated from human degenerated IVD may be divided into two groups according to the expression of mesenchymal stem cell (MSC) surface markers: The high expression of MSC surface markers group (H-NPSCs) was highly positive for CD29, CD44, CD73, CD90 and $\mathrm{CD} 105$ at rates $>95 \%$, and the low expression of MSC markers surface markers group (L-NPSCs), with the expression of CD29 and CD105 exhibiting individual variability, however, all at rates $<95 \%$. The donors for H-NPSCs were aged $<20$ years, while the majority of donors for L-NPSCs were aged $>25$ years, with one exception aged $<20$ years. The results highlighted that the low expression of MSC surface markers in NPSCs from aged and degenerated NP tissues were associated with a low rate of proliferation and reduced differentiation potential, as well as downregulation
\end{abstract}

Correspondence to: Dr Jiaqing Zhang, Department of Biochemistry and Molecular Biology, School of Preclinical Medicine, Jinan University, 601 Huangpu Avenue West, Guangzhou, Guangdong 510632, P.R. China

E-mail: zhangjiaqing@jnu.edu.cn

*Contributed equally

Key words: nucleus pulposus progenitor/stem cells, degenerated intervertebral disc, regenerative potential, phenotypic markers of the NP progenitor marker Tie2 and higher expression of NP cell-specific markers. These findings demonstrated that the regenerative potential of human NPSCs declines with ageing and degeneration of the IVD.

\section{Introduction}

Degenerative intervertebral disc (IVD) disease and associated chronic lower back pain constitute a major health concern and have a significant socioeconomic burden $(1,2)$. The IVD consists of a gel-like nucleus pulposus (NP) surrounded by the annulus fibrosus (AF) and thin hyaline cartilaginous end plates, and provides a connection between the vertebral bodies. During IVD degeneration, the disc undergoes a shift in balance from anabolism to catabolism, including decreased production of aggrecan and type II collagen, increased production of matrix metalloproteinases and inflammatory factors, and increased cytokine production (3-5). These changes are mediated by disturbances in the function of cells residing in the disc (6,7). It is well established that the NP contains NP resident stem/progenitor cells (NPSCs), which serve a major role in maintaining cellular homeostasis and regeneration following aging and injury (8). NPSCs have been found to maintain multipotent and self-renewal potential when cultured in vitro $(9,10)$, and the resilience of the IVD cell population may be ensured if the relevant stem cell populations give rise to differentiated progeny over a person's lifetime $(11,12)$. Although the cellular identity of NPSCs that reside in the degenerated IVD remains poorly defined, numerous studies have reported that stem/progenitor cells expressing mesenchymal stem cell (MSC) markers have been isolated successfully from the degenerated IVD (13-15). However, the data on NPSCs regarding phenotype signature and biological capacity during IVD degeneration remain controversial $(16,17)$.

Since the discovery and characterization of multipotent MSCs in the bone marrow, identification of the NPSCs has also principally depended on the MSC markers primarily developed for bone marrow-derived MSCs (BMMSCs), despite their tissue specificity. 
The present study was undertaken to explore the hypothesis that the regenerative potential of NPSCs isolated from patients with degenerative IVD diseases declines with ageing and IVD degeneration. The expression of MSC surface markers in NPSCs from human degenerated discs was investigated in order to determine their proliferation capacity and multilineage differentiation potential, with the hope of elucidating the mechanism underlying IVD degeneration and developing novel treatment strategies.

\section{Materials and methods}

Sample collection. A total of 10 NP tissue samples were obtained from patients who underwent microendoscopic discectomy for degenerative spine diseases. NP tissues were stored in PBS solution under sterile conditions. The patient and sample details are summarized in Table I. All the procedures performed for the present study were approved by the medical ethics committee of Jinan University (Guangzhou, China). Specific informed consent was obtained in all cases.

Isolation of MSC-like cells from NP tissues. To distinguish gelatinous NP tissues from AF regions, the collection of gelatinous NP tissue from surgically removed human NP tissues was performed using a stereoscopic microscope. An explant culture method was employed to isolate NPSCs from NP tissue as previously described (18). In brief, NP tissues were cut into $1 \mathrm{~mm}^{3}$ pieces and incubated at $37^{\circ} \mathrm{C}$ in a $5 \%$ $\mathrm{CO}_{2}$ incubator without culture medium for $2 \mathrm{~h}$ to allow tissue attachment. Complete culture medium containing Dulbecco's Modified Eagle's Medium (DMEM)/F12 supplemented with $20 \%$ fetal bovine serum (FBS), 1\% L-glutamine and 1\% penicillin-streptomycin (all from Invitrogen; Thermo Fisher Scientific, Inc., Waltham, MA, USA) was added to the tissue culture dishes and incubated for an additional 17 days. The primary cells that had migrated out of the NP tissues and attached to dishes were passaged by a 2 -min treatment with $0.25 \%$ trypsin and $0.02 \%$ EDTA at $37^{\circ} \mathrm{C}$. The medium was replaced every 2 days. Cells were further passaged when they reached 80-90\% confluence. All cells used in the experiments were at passages 2-4.

\section{Flow cytometry assay}

Analysis of cell surface marker expression. A flow cytometry assay was used to identify the expression of specific surface markers in NPSCs. A total of $\sim 1 \times 10^{6}$ cells were re-suspended in PBS (containing 5\% FBS and 1\% BSA) to produce a single-cell suspension, then incubated with fluorescein isothiocyanate-conjugated antibodies against human CD29 (1:100 dilution, cat. no. ab150002, mouse monoclonal, Abcam, Cambridge, MA, USA), CD44 (1:100 dilution, cat. no. ab46793, mouse monoclonal, Abcam), CD73 (1:100 dilution, cat. no. ab106697, mouse monoclonal, Abcam), CD90 (1:50 dilution, cat. no. ab134360, mouse monoclonal, Abcam), CD105 (1:20 dilution, cat. no. ab53321, mouse monoclonal, Abcam), CD11b (1:20 dilution, cat. no. ab28101, mouse monoclonal, Abcam), CD14 (1:20 dilution, cat. no. ab91146, mouse monoclonal, Abcam), CD24 (1:20 dilution, cat. no. ab30350, mouse monoclonal, Abcam), CD34 (1:100 dilution, cat. no. ab187284, mouse monoclonal,
Abcam), CD45 (1:100 dilution, cat. no. ab157309, mouse monoclonal, Abcam) and HLA-DR (1:50 dilution, cat. no. ab59476, mouse monoclonal, Abcam) and appropriate isotype control-mouse IgG2A-FITC (1:100; Miltenyi Biotech, Bergisch Gladbach, Germany) or IgG1-PE (1:100; Molecular Probes, Life Technologies, Inc.; Thermo Fisher Scientific, Waltham, MA, USA).

The samples were incubated in the dark at $4^{\circ} \mathrm{C}$ for $30 \mathrm{~min}$. Finally, labelled cells were washed three times with PBS and surface marker expression was detected using flow cytometry (BD Biosciences, Franklin Lakes, NJ, USA).

Cell cycle analysis. Cells were harvested as previously described (18), and washed twice with PBS containing $2 \%$ FBS. The cells were then fixed in pre-chilled absolute ethanol at $4^{\circ} \mathrm{C}$ for $>1 \mathrm{~h}$. An equal amount of PBS was added twice for washing. A total of $100 \mu \mathrm{l}$ RNaseA was added at $37^{\circ} \mathrm{C}$ for $30 \mathrm{~min}$, followed by the addition of propidium iodide (PI) (Invitrogen; Thermo Fisher Scientific, Inc.) at $4^{\circ} \mathrm{C}$ in the dark for $30 \mathrm{~min}$. Cell cycle was analyzed by flow cytometry (BD Biosciences), using BD FACSuite ${ }^{\mathrm{TM}}$ software.

Apoptosis analysis. Apoptosis and cell death were assessed by flow cytometry using Annexin V-FITC (Invitrogen; Thermo Fisher Scientific, Inc.) and PI. Cultured cells were detached, suspended in PBS and stained with Annexin V-FITC and PI according to the manufacturer's protocol. Apoptotic cells were identified as an Annexin V-positive/PI-negative population. Analysis was performed using the CellQuest software Pro (BD Biosciences).

\section{Cell proliferation and viability analysis}

Growth curves and population doubling time. Cells at passage 3 were seeded at a density of $3 \times 10^{4}$ cells/well in a 24 -well culture plate. The cells were harvested by trypsinization from each well as previously described (18), and a duplicate using a hemocytometer to count the cells, every day for a total of 13 days. The growth curve was plotted with the cell culture time as the horizontal axis and the cell number as the vertical axis. The cell population doubling time was calculated as: DT $=\mathrm{t}(\log 2) /\left(\log \mathrm{N}_{\mathrm{t}}-\log \mathrm{N}_{0}\right)$ and the results were analyzed by GraphPad Prism 5 software (GraphPad Software, Inc., La Jolla, CA, USA).

Cell viability. Cell viability was measured with a Cell Counting Kit-8 (CCK-8; Dojindo Molecular Technologies, Inc., Kumamoto, Japan) according to the manufacturer's protocol. Briefly, cells were seeded at a density of $3 \times 10^{4}$ cells/well in a 24-well cell culture plate. Proliferation rates were evaluated at day $1,3,5,7,9,11$ and 13 . The absorbance at $450 \mathrm{~nm}$ was measured with a microplate reader (Bio-Rad Laboratories, Inc., Hercules, CA, USA). A colorimetric assay was used to create cell viability curves using the mean results from three independent experiments.

Detection of the expression of marker genes with reverse transcription-quantitative polymerase chain reaction ( $R T-q P C R)$. The gene expression of NP cell phenotypic markers, NP progenitor cell-specific gene and pluripotent stem cell markers was analyzed via RT-qPCR. mRNA was isolated from NPSCs 
Table I. Characteristics of the patients enrolled in the study.

\begin{tabular}{|c|c|c|c|c|c|c|}
\hline Case no. & Age (years) & Sex & Symptoms & Diagnosis & Disc level & $\begin{array}{c}\text { Pfirrmann } \\
\text { grade }\end{array}$ \\
\hline 1 & 18 & M & BP-RP & Lumbar disc herniation & $\mathrm{L} 4 / 5$ & II \\
\hline 2 & 16 & $\mathrm{~F}$ & BP-RP & Lumbar disc herniation & $\mathrm{L} 5 / \mathrm{S} 1$ & II \\
\hline 3 & 34 & $\mathrm{~F}$ & $\mathrm{BP}$ & Lumbar disc herniation & $\mathrm{L} 5 / 6$ & IV \\
\hline 4 & 25 & $\mathrm{M}$ & BP & Lumbar disc herniation & $\mathrm{L} 4 / 5$ & III \\
\hline 5 & 28 & $\mathrm{~F}$ & BP & Lumbar disc herniation & $\mathrm{L} 5 / \mathrm{S} 1$ & III \\
\hline 6 & 42 & M & BP-RP & Lumbar disc herniation & $\mathrm{L} 5 / \mathrm{S} 1$ & IV \\
\hline 7 & 19 & $\mathrm{~F}$ & BP-RP & Lumbar disc herniation & $\mathrm{L} 4 / 5$ & III \\
\hline 8 & 49 & $\mathrm{~F}$ & $\mathrm{BP}$ & Lumbar disc herniation & $\mathrm{L} 4 / 5$ & IV \\
\hline 9 & 41 & M & BP & Lumbar disc herniation & $\mathrm{L} 5 / \mathrm{S} 1$ & III \\
\hline 10 & 38 & $\mathrm{~F}$ & BP & Lumbar disc herniation & $\mathrm{L} 5 / \mathrm{S} 1$ & III \\
\hline
\end{tabular}

M, male; F, female; BP, back pain; RP, radicular pain.

Table II. Primers used for this study.

\begin{tabular}{lll}
\hline Gene name & \multicolumn{1}{c}{ Forward primer (5' to 3') } & Reverse primer (5' to 3') \\
\hline SOX9 & AGCGAACGCACATCAAGAC & CTGTAGGCGATCTGTTGGGG \\
ACAN & TCGAGGACAGCGAGGCC & TCGAGGGTGTAGCGTGTAGAGA \\
Tie 2 A 1 & GGCAATAGCAGGTTCACGTACA & CGATAACAGTCTTGCCCCACTT \\
NANOG & AATCACTATGAGGCTTGGCAACAT & GCGTCTCACAGGTCCAGGAT \\
GAPDH & GATTTGTGGGCCTGAAGAAA & CAGATCCATGGAGGAAGGAA \\
& GAGAAGGAGAAGCTGGAGCA & AATAGAACCCCCAGGGTGAG \\
\hline
\end{tabular}

using the TRIzol (Invitrogen; Thermo Fisher Scientific, Inc.) extraction method. cDNA synthesis was performed using reverse transcriptase SuperScript III according to the manufacturer's protocol (Invitrogen; Thermo Fisher Scientific, Inc.). All polymerase chain reactions were conducted using ABI Prism 7500 (Applied Biosystems; Thermo Fisher Scientific, Inc.) and gene expression levels were quantified using SYBR Green (Invitrogen; Thermo Fisher Scientific, Inc.). The data were normalized to glyceraldehyde-3-phosphate dehydrogenase $(G A P D H)$ values. Relative gene expression levels were calculated by the comparative $\mathrm{Cq}$ method. The primers used (synthesized by Invitrogen; Thermo Fisher Scientific, Inc.) are listed in Table II.

Multilineage differentiation potential. Osteogenic and adipocytic differentiation of subconfluent cells was induced by induction media for 21 days, as previously described (18). For chondrogenesis, a total of $3.5 \times 10^{5}$ cells were centrifuged at $200 \mathrm{xg}$ at room temperature for $5 \mathrm{~min}$ to form a three-dimensional aggregate in a $15-\mathrm{ml}$ conical tube, then incubated with chondrogenic media containing DMEM/F12 supplemented with 10\% ITS, 10-7 M dexamethasone, $1 \mu \mathrm{M}$ ascorbate-2-phosphate, $1 \%$ sodium pyruvate and $10 \mathrm{ng} / \mathrm{ml}$ transforming growth factor- $\beta 1$ (TGF- $\beta 1$ ) (Cyagen,
Guangzhou, China) for 21 days. The induced medium was replaced every 2-3 days.

For Alizarin Red S (ARS) staining, cells were fixed in $4 \%$ paraformaldehyde (PFA) at room temperature for $10 \mathrm{~min}$ and stained at room temperature for 5-10 min with $40 \mathrm{mM}$ ARS solution (Solarbio, Inc., Beijing, China). Oil red O solution (Solarbio, Inc.) was used to stain intracellular lipid vacuoles at room temperature for 5-10 min after fixation with PFA. To identify chondrogenic differentiation, the cell pellets were stained with Alcian blue (Solarbio, Inc.) at room temperature for 5-10 min, fixed in PFA, as described previously, then frozen in OCT freezing medium (Sakura Finetek USA, Inc., Torrance, CA, USA) and sectioned into 5- $\mu \mathrm{m}$ slices.

Further quantitative analysis was performed using ImageJ software (National Institutes of Health, Bethesda, MA, USA).

Statistical analysis. The data are presented as mean \pm standard deviation. One-way analysis of variance (ANOVA) was conducted to analyze the differences among different cell types. $\mathrm{P}<0.05$ was considered to indicate a statistically significant difference. If ANOVA indicated a significant difference between the groups, the difference was evaluated using the least significant difference test. 

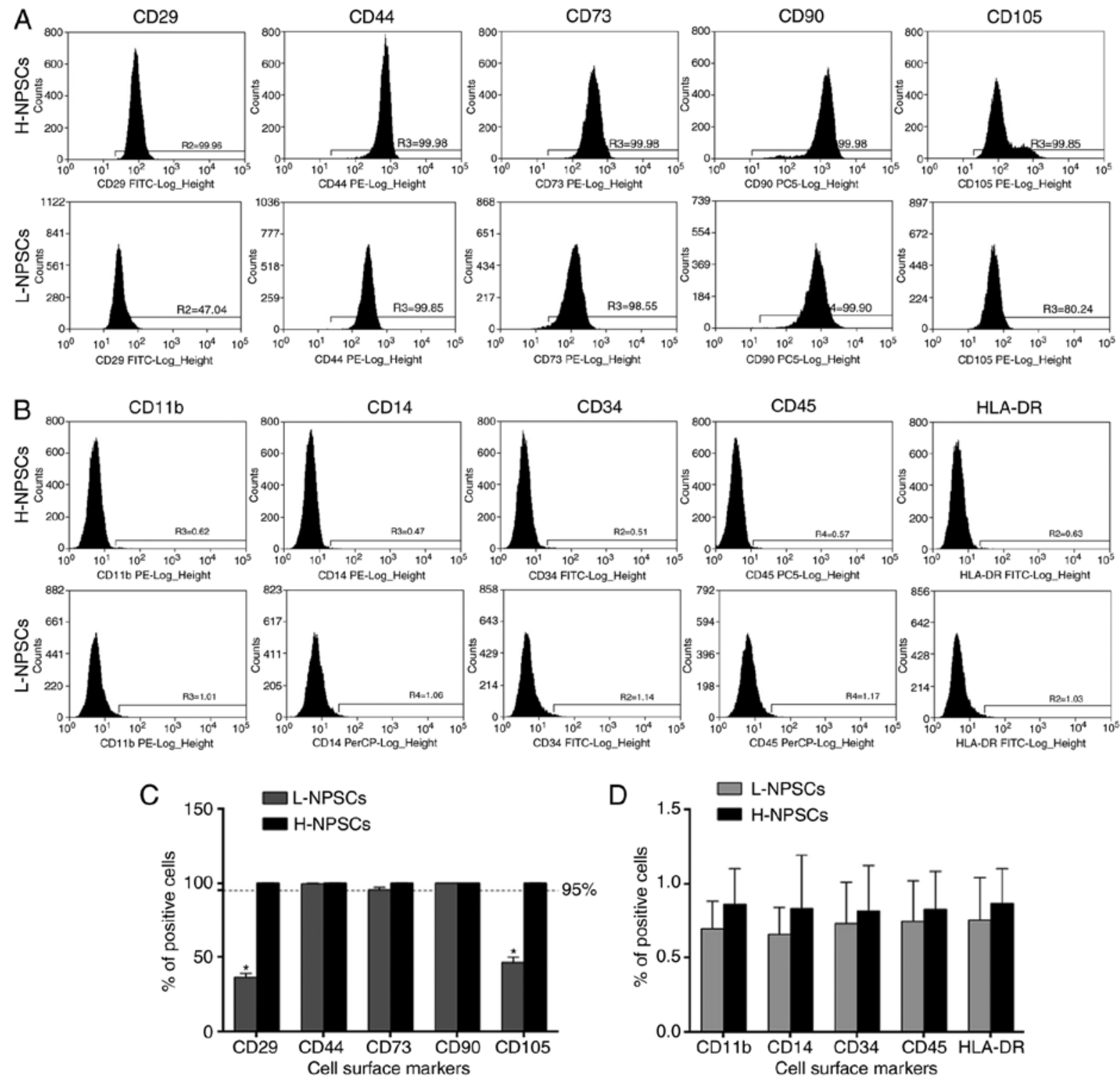

Figure 1. Flow cytometric analysis of the expression of surface markers. (A) H-NPSCs and L-NPSCs were positive for the MSC surface markers CD29, CD44, CD73, CD90 and CD105. (B) H-NPSCs and L-NPSCs were negative $(<1 \%)$ for the hematopoietic stem cell surface markers CD11b, CD14, CD34, CD45 and HLA-DR. (C) Comparative analysis of positivity for MSC surface markers between L-NPSCs and H-NPSCs. (D) Comparative analysis of positivity for hematopoietic stem cell surface markers between L-NPSCs and H-NPSCs. The data are expressed as means \pm standard deviation, ${ }^{*} \mathrm{P}<0.05$ vs. H-NPSCs. NP, nucleus pulposus; MSC, mesenchymal stem cell; H-NPSC, high expression of MSC surface markers group; L-NPSC, low expression of MSC surface markers group.

\section{Results}

Flow cytometry assay

Expression of cell surface markers. The flow cytometry results demonstrated that NPSCs from 2 young donors were highly positive for the expression markers CD29, CD44, CD73, CD90 and $\mathrm{CD} 105$ at rates of $>95 \%$ (Fig. 1A and C), and negative for CD11b, CD14, CD34, CD45 and HLA-DR (<1\%, Fig. 1B and D), which fulfilled the ISCT requirements for MSC definition and were classified as the high expression of MSC surface markers group (H-NPSCs). In NPSCs from the remaining 8 donors that were aged $>25$ years, with one exception aged $<20$ years, the expression of CD29 and CD105 exhibited interindividual variability; however, all rates were $<95 \%$. The expression rates of CD73,CD44 and CD90 were $>95 \%$ and the expression of CD11b, CD14, CD34, CD45 and HLA-DR were negative $(<1 \%)$, thus classified as the low expression of MSC surface markers group (L-NPSCs). Although H-NPSCs and L-NPSCs expressed the NP cell surface marker CD24 at low rates, the rate in L-NPSCs was significantly higher compared with that in H-NPSCs $(\mathrm{P}<0.05$, Fig. 2). As the MSC positive antigens were expressed at diverse levels for NPSCs isolated from different degenerated disc samples, it was necessary to explore further the possible variability in NPSC biological characteristics with regards to the cell cycle, apoptosis, proliferative capacity, differentiation potential and NP-specific gene expression.

Cell cycle and apoptosis analysis. A significant difference was detected between H-NPSCs and L-NPSCs in the $\mathrm{G}_{0} / \mathrm{G}_{1}$ phases of the cell cycle $(\mathrm{P}<0.05)$. The percentage of H-NPSCs in the $\mathrm{G} 2 / \mathrm{M}$ and $\mathrm{S}$ phases $\left(\mathrm{G}_{2} / \mathrm{M}+\mathrm{S}\right)$ was $\sim 1.7$-fold higher compared with that of the L-NPSC group $(\mathrm{P}<0.05$, Fig. 3$)$. This result demonstrated a reduced proliferative activity of L-NPSCs 
A

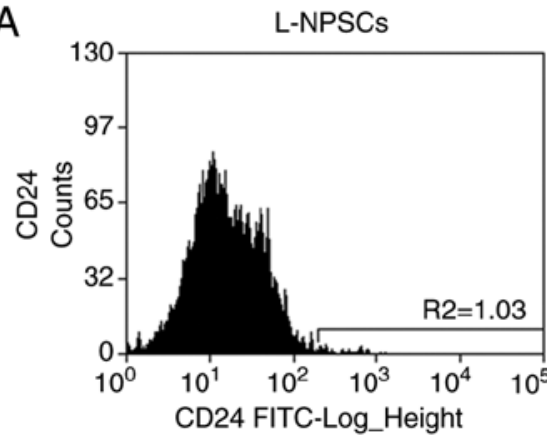

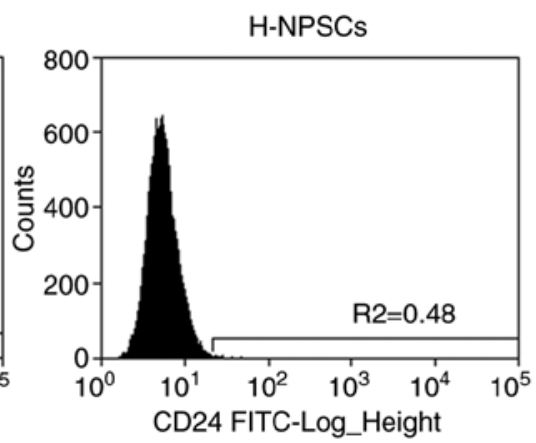

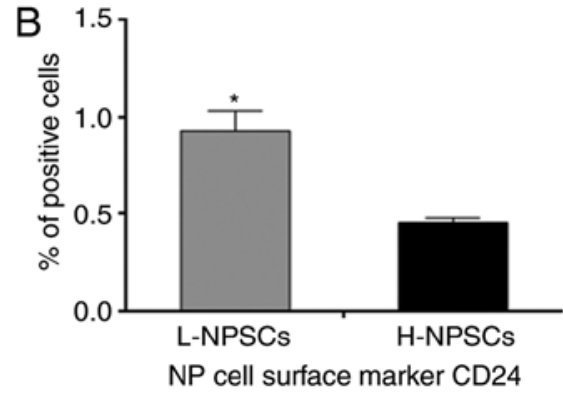

Figure 2. (A) Flow cytometric analysis of NP cell surface marker CD24 expression in L-NPSCs and H-NPSCs. (B) Comparative analysis of positivity for CD24 between L-NPSCs and H-NPSCs. The data are expressed as means \pm standard deviation, "P $<0.05$ vs. H-NPSCs. NP, nucleus pulposus; MSC, mesenchymal stem cell; H-NPSC, high expression of MSC surface markers group; L-NPSC, low expression of MSC surface markers group.
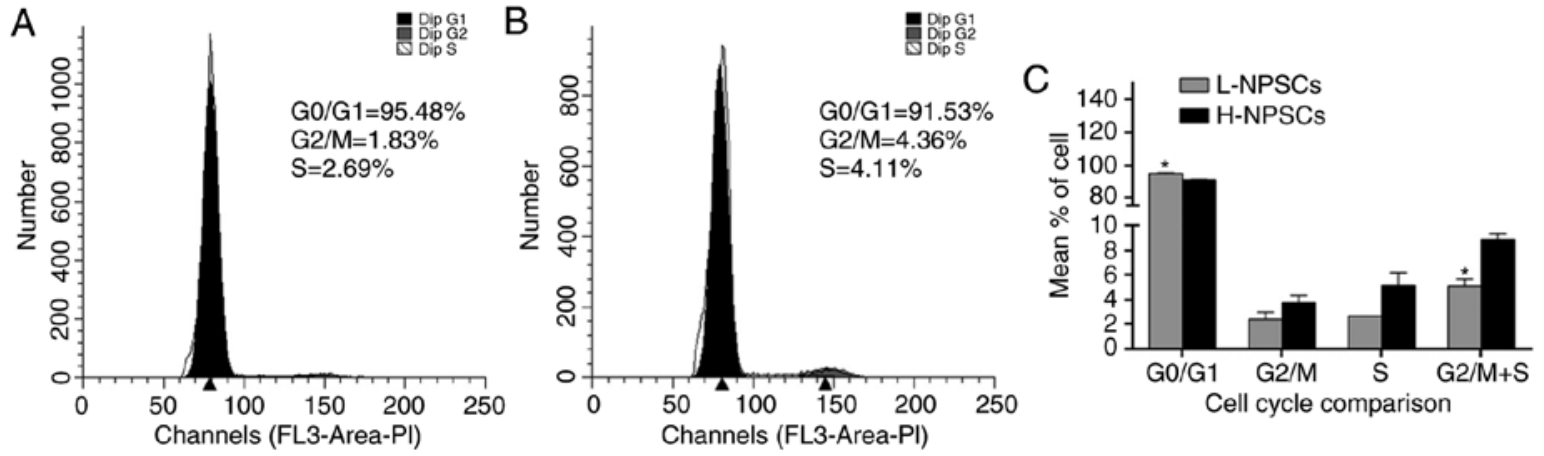

Figure 3. The cell cycles of (A) L-NPSCs and (B) H-NPSCs were assessed at Passage 3 with flow cytometry. (C) Comparison of cell cycle analysis between L-NPSCs and H-NPSCs. The data are expressed as means \pm standard deviation, $n=3, " P<0.05$ vs. H-NPSCs. H-NPSC, high expression of MSC surface markers group; L-NPSC, low expression of MSC surface markers group; NP, nucleus pulposus; MSC, mesenchymal stem cell.
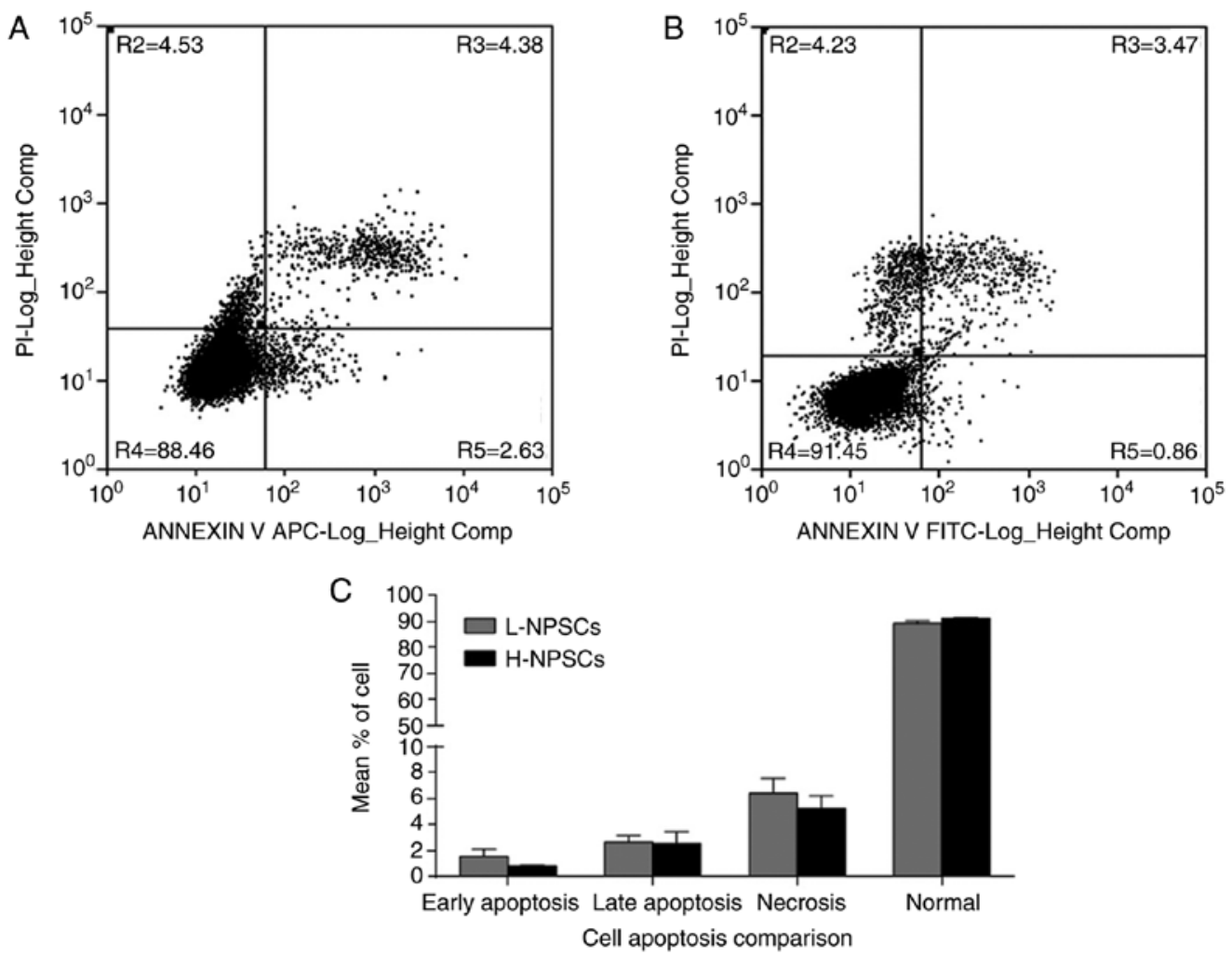

Figure 4. The apoptosis of (A) L-NPSCs and (B) H-NPSCs was assessed at Passage 3 with flow cytometry. (C) Comparative analysis of apoptosis between L-NPSCs and H-NPSCs. The data are expressed as means \pm standard deviation. H-NPSC, high expression of MSC surface markers group; L-NPSC, low expression of MSC surface markers group; NP, nucleus pulposus; MSC, mesenchymal stem cell. 


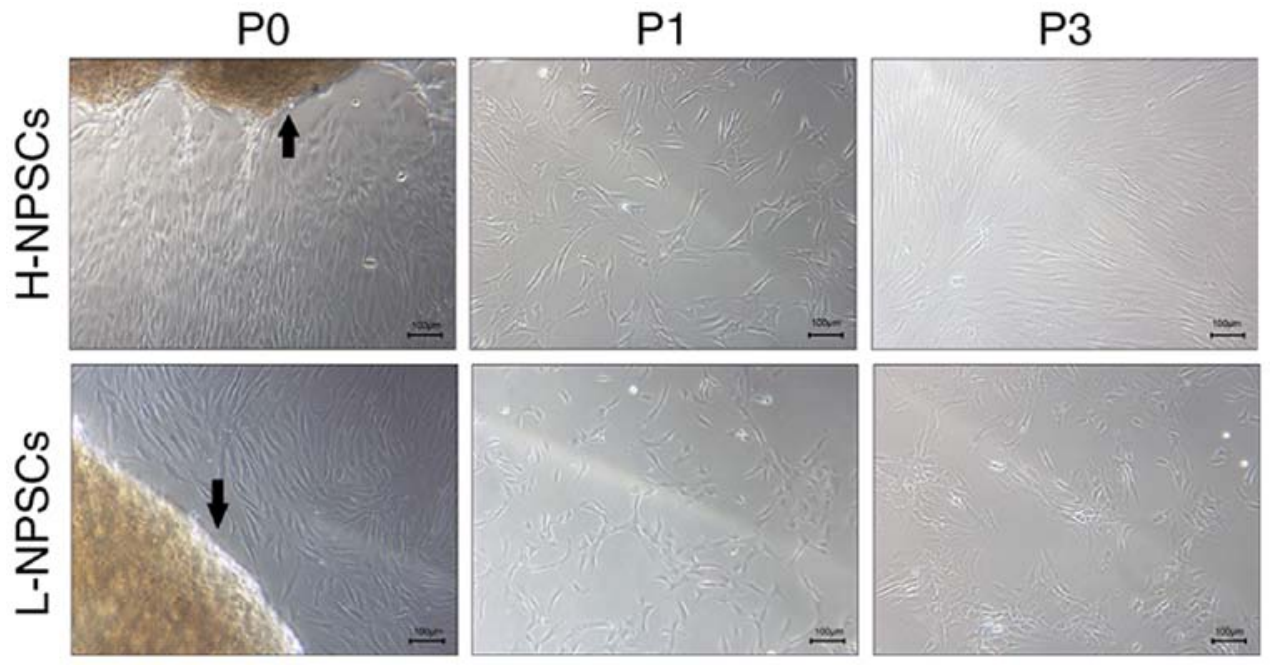

Figure 5. Morphology of H-NPSCs and L-NPSCs cultured ex vivo. Scale bar, $100 \mu \mathrm{m}$. The arrows in the figure indicate the pieces of NP tissue. P, passage; NP, nucleus pulposus; MSC, mesenchymal stem cell; H-NPSC, high expression of MSC surface markers group; L-NPSC, low expression of MSC surface markers group.
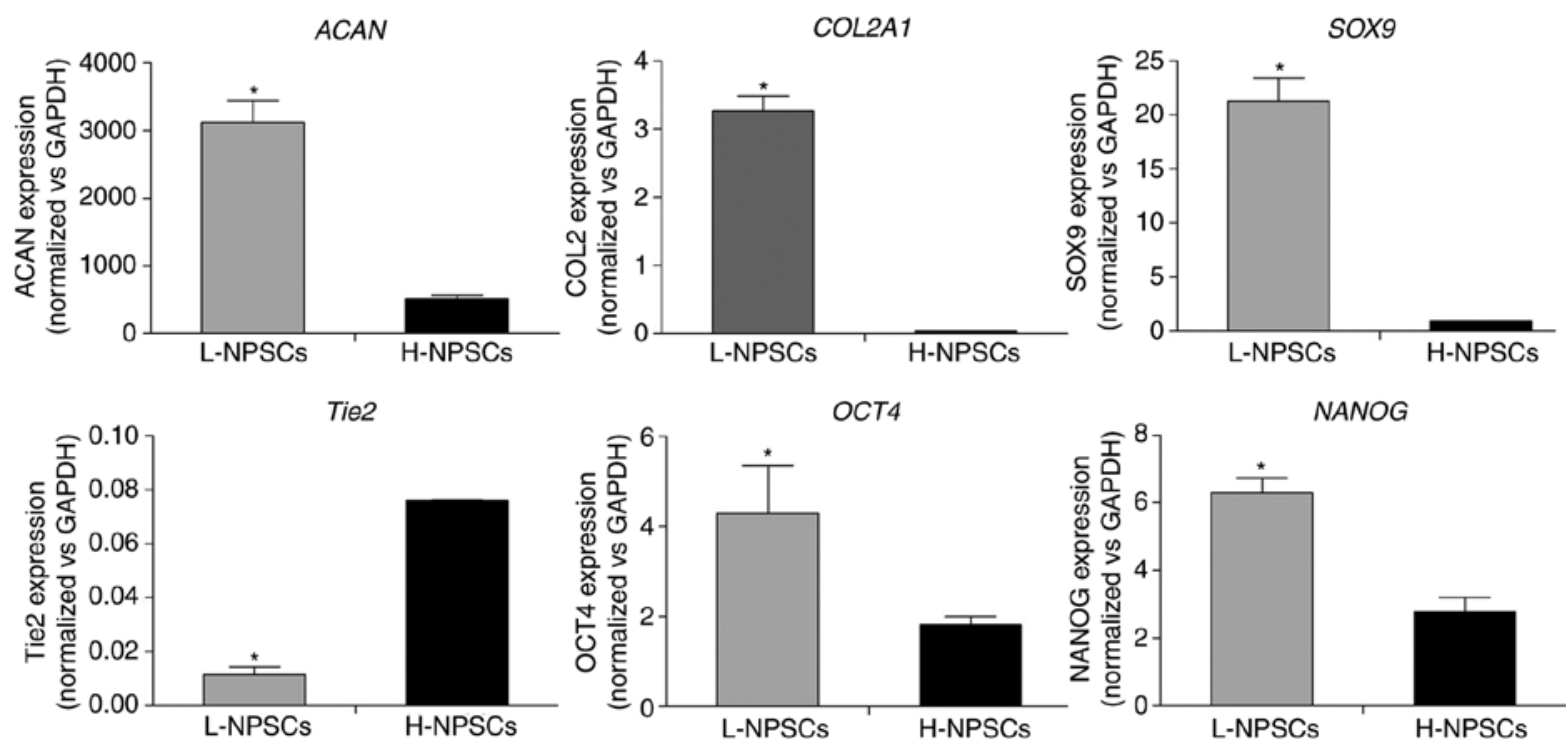

Figure 6. Reverse transcription-quantitative polymerase chain reaction for ACAN, COL2A1, SOX9, Tie2, OCT4 and NANOG was performed and data are presented as normalized to GAPDH. The data are expressed as mean \pm standard deviation. " $\mathrm{P}<0.05$ vs. H-NPSCs. H-NPSC, high expression of MSC surface markers group; NP, nucleus pulposus; MSC, mesenchymal stem cell.

compared with H-NPSCs. Similarly, the flow cytometry results demonstrated that the rates of cell apoptosis and necrosis were higher in L-NPSCs when compared with those in H-NPSCs (Fig. 4).

Morphology. Cell morphology was assessed using phase-contrast microscopy. At the primary passage (P0-P1), the majority of cells among H-NPSCs and L-NPSCs exhibited a typical spindle shape, with a more heterogeneous cell morphology observed in the L-NPSCs culture (Fig. 5). Morphological changes of cells were displayed during passaging; H-NPSCs maintained a homogeneous population with typical MSC-shaped morphology; however, L-NPSCs exhibited a heterogeneous morphology, with the appearance of a varied proportion of polygonal or digital-shaped cells
(Fig. 5). Additionally, the L-NPSC culture required a longer time to achieve confluence compared with H-NPSCs.

$R T$ - $q P C R$ analysis. The results in Fig. 6 demonstrated that the expression levels of SOX9, COL2A1 and ACAN in L-NPSCs were 6-, 86- and 22-fold higher, respectively, compared with those in H-NPSCs $(\mathrm{P}<0.05)$. The opposite trend was observed for the NP progenitor cell-specific marker Tie2, which demonstrated a significantly higher expression levels in H-NPSCs compared with that in L-NPSCs (Fig. 6, P<0.05). These indices consistently demonstrated that part of cells in the L-NPSCs group may have differentiated into NP cells.

Notably, the expression of OCT4 and NANOG, as transcription factors mediating self-renewal and an undifferentiated state (17), were typically higher in L-NPSCs compared with 

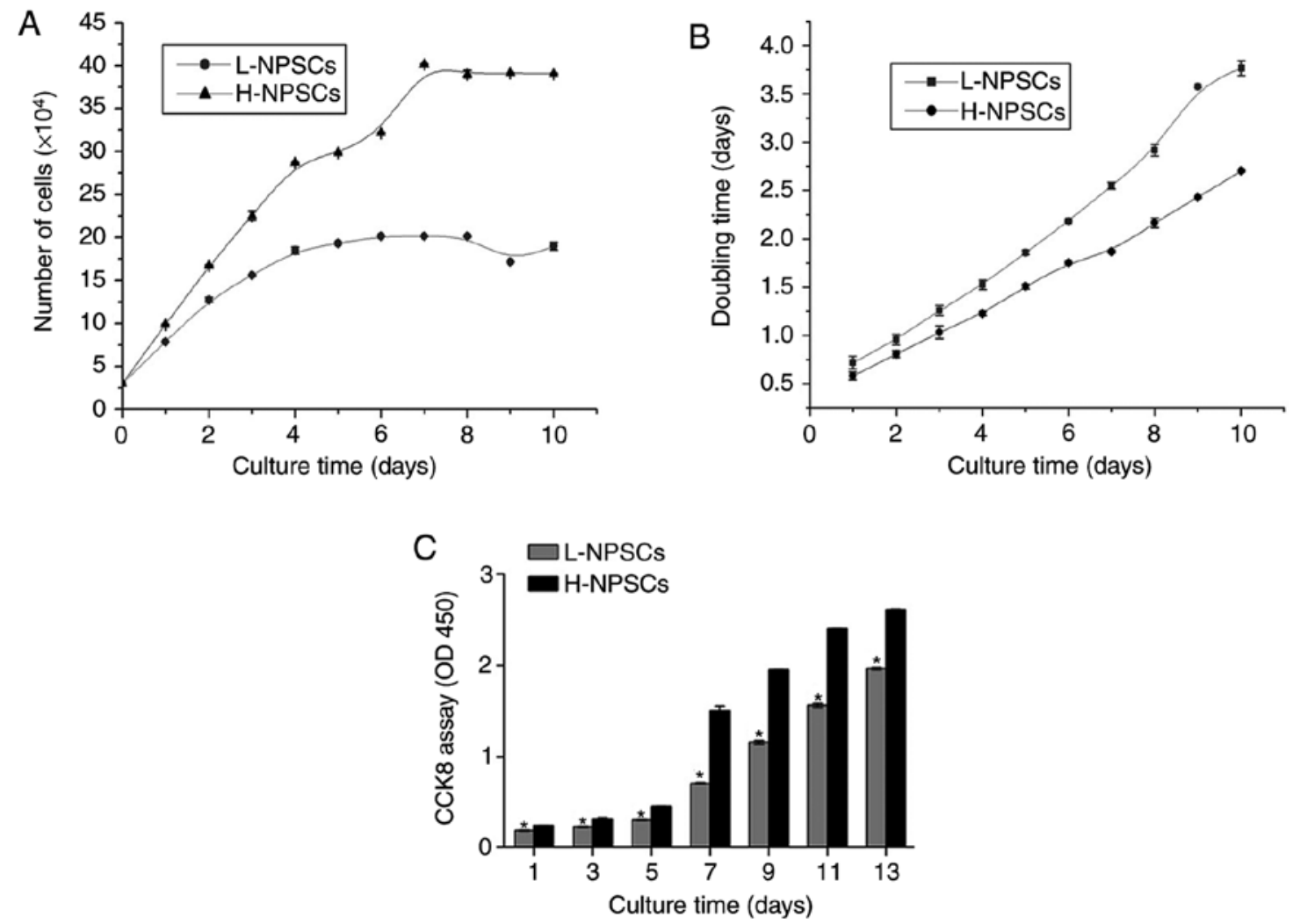

Figure 7. In vitro proliferation ability of L-NPSCs and H-NPSCs at passage 3. (A) Absolute growth rate. (B) Doubling time. (C) The viability of L-NPSCs and H-NPSCs was analyzed by Cell Counting Kit-8 on days 1, 3, 5, 7, 9, 11 and 13 . The data are expressed as mean \pm standard deviation. ${ }^{*}<<0.05$ vs. H-NPSCs. H-NPSC, high expression of MSC surface markers group; L-NPSC, low expression of MSC surface markers group; NP, nucleus pulposus; MSC, mesenchymal stem cell.

H-NPSCs. These findings suggested that the expression of pluripotency markers and NP-specific markers were associated with the MSC immunophenotypic pattern in NP-derived stem/progenitor cells.

Cell proliferation and viability analysis. In order to compare the proliferative capacity between H-NPSCs and L-NPSCs, a growth curve was plotted and the doubling time was calculated (Fig. 7). According to the growth curve, H-NPSCs accelerated rapidly during days 3-7, and slowed down thereafter. L-NPSCs continued to grow for 3-4 days, and reached a cell growth plateau at days 6-8 (Fig. 7A). The doubling time was calculated based on the results illustrated in Fig. 7A, which demonstrated that L-NPSCs had a much longer doubling time compared with H-NPSCs (Fig. 7B).

The viability of H-NPSCs and L-NPSCs was assessed with the CCK-8 method (Fig. 7C). The optical density (OD) values of H-NPSCs were significantly higher compared with L-NPSCs, which were consistent with the results of population doubling time and growth curves. According to the abovementioned results, the growth ability of H-NPSCs was markedly higher compared with that of L-NPSCs, indicating that cells with higher intensity of MSC surface marker expression exhibited a more prominent proliferative capacity.

Multilineage differentiation potential. Assays evaluating differentiation into osteogenic, adipogenic and chondrogenic lineages were performed with specific staining. After 3 weeks of osteogenic induction, H-NPSCs exhibited an extensive mineralized matrix, as demonstrated by strong Alizarin red staining. Conversely, L-NPSCs exhibited few calcium deposits when stained by Alizarin red (Fig. 8A). Further quantitative analysis revealed that the percentage of the positively stained area was significantly lower in L-NPSCs compared with that in H-NPSCs. The examined NPSCs displayed intracellular lipid vacuoles after 21 days of adipogenic induction, which were visualized with Oil red O staining. However, the H-NPSCs appeared to form an increased number of fat drops compared with the L-NPSCs (Fig. 8B). Quantification was performed by determining the percentage of the area that contained Oil Red O-stained lipid vacuoles, and the percentage in L-NPSCs was significantly lower compared with that in H-NPSCs. Similarly, the amount of Alcian blue staining used to determine chondrogenic differentiation was higher in H-NPSCs compared with that in L-NPSCs (Fig. 8C).

\section{Discussion}

The identification of stem/progenitor cells within human IVDs indicated the presence of a natural repair mechanism within the disc, which may be activated for regeneration (19).

The characterization of the NPSCs was principally based on the surface markers primarily developed for BMMSCs, although there is a recent report on the existence of an NP progenitor-specific marker (20). A number of studies have reported that the markers that are positive in BMMSCs, 
A

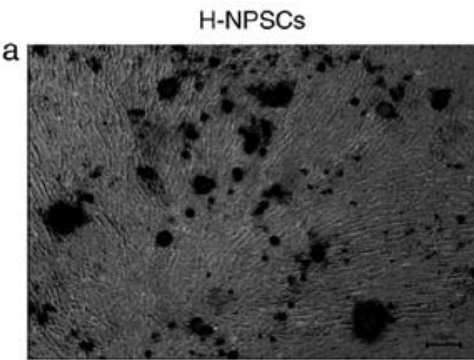

$\mathrm{B}$ a

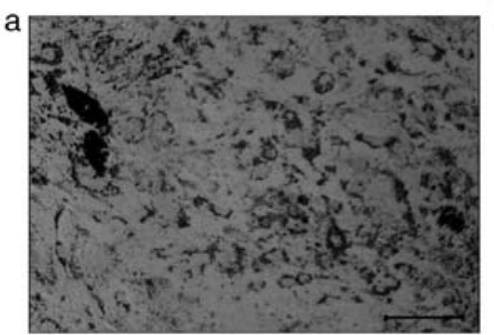

$\mathrm{C}$ a

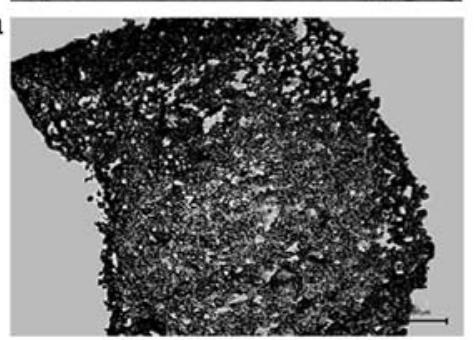

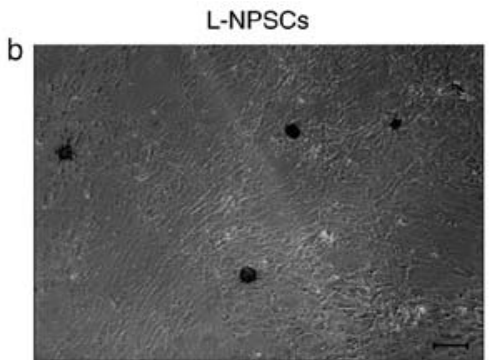
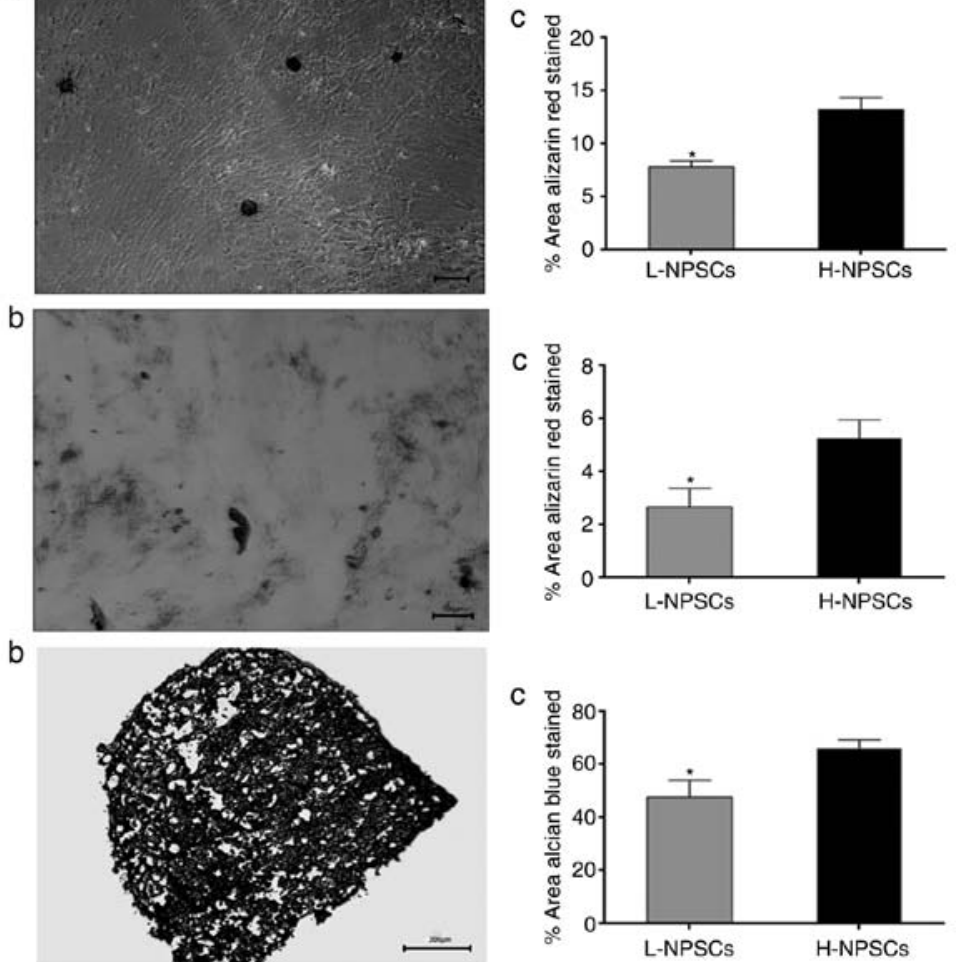

Figure 8. Multipotent differentiation capacity of L-NPSCs and H-NPSCs. (A) Osteogenic differentiation capacity of L-NPSCs and H-NPSCs. (B) Adipogenic differentiation capacity of L-NPSCs and H-NPSCs. (C) Chondrogenic differentiation capacity of L-NPSCs and H-NPSCs. (a) L-NPSCs, (b) H-NPSCs and (c) comparative analyses. The data are expressed as means \pm standard deviation. ${ }^{*} \mathrm{P}<0.05$ vs. H-NPSCs. H-NPSC, high expression of MSC surface markers group; L-NPSC, low expression of MSC surface markers group; NP, nucleus pulposus; MSC, mesenchymal stem cell.

including CD29, CD44, CD73, CD90 and CD105, are also positive in NPSCs from degenerated IVDs; and CD34 and CD45, which are negative in BMMSCs, are also negative in NPSCs (13-17). However, in the present study, the expression of CD29 and CD105 were significantly downregulated, while CD44, CD73 and CD90 were expressed at rates $>95 \%$ in H-NPSCs compared with that in L-NPSCs, which expressed the examined markers at rates $<95 \%$ and failed to fulfill the ISCT requirement for MSC definition.

CD105 is a receptor for transforming growth factor- $\beta$ (TGF- $\beta$ ) and is associated with cell adhesion and migration (21). The decreased expression of CD105 may signify declined capacities of homing and migration (21). CD29 is an anchorage protein involved in cell adhesion and migration, and its expression indicates cell populations with a higher migratory capacity (22). Although the low intensity of CD105 expression was reported by Blanco et al (13), this result was different from previous reports, which demonstrated that MSC-like cells from degenerated NP tissues displayed a MSC marker profile that was highly positive for $\mathrm{CD} 73, \mathrm{CD} 90, \mathrm{CD} 105$, CD44, CD56 and CD146, and negative for CD45 $(23,24)$. This may be attributed to a different quality of the NP tissues obtained from surgical specimens. In the present study, the specimens used were not any NP tissues, but specifically the herniated NP tissues removed during endoscopic discectomy. Hegewald et al (25) reported that cells harvested from herniated NP tissue and grown in a 3D culture system possess limited regenerative potential compared with cells from a degenerated but contained NP compartment. Accordingly, we hypothesized that the NP tissues L-NPSCs were isolated from may have undergone more extensive degeneration compared with H-NPSCs residing in NP tissues, and the activities of the resident NPSCs would be altered accordingly.

The immunophenotypic data were supported by the morphologic results: L-NPSCs exhibited a heterogeneous morphology with the appearance of a varied proportion of polygonal or digital-shaped cells, even at the 3rd passage; however, H-NPSCs consistently exhibited a typical MSC morphology.

The expression of NP-specific progenitor marker and NP cell marker Tie2, also referred to as CD202b, is a cellular membrane receptor tyrosine kinase of the Tie family (23). Tie 2 has been identified as a marker of NP precursor cells that were found to exhibit multipotency and self-renewal capacity in animal and human NP $(23,26)$. The results of the present study demonstrated that L-NPSCs and H-NPSCs expressed a low level of Tie2; however, L-NPSCs displayed a significantly lower level compared with H-NPSCs, suggesting that the number of progenitor cells among L-NPSCs was decreased. This was also supported by the immunophenotypic results, which demonstrated an upregulation of the mature NP cell marker CD24 in L-NPSCs.

Native adult NP cells are conventionally described as 'chondrocyte-like' and characterized through their rounded morphology and expression of classic chondrogenic markers, including SOX9, type II collagen (COL2Al) and aggrecan $(A C A N)$ (27). SOX9 is the major regulator of the chondrocytic phenotype and serves as a potent promoter of $C O L 2 \mathrm{Al}$ 
gene expression, which is almost exclusively produced in the chondrocyte. The proteoglycan aggrecan is also a characteristic gene product of chondrocytes (28). These markers are characteristic of and relevant to healthy NP cells (29).

Gene expression analysis for the classic chondrogenic marker genes $A C A N, C O L 2 A 1$ and $S O X 9$ was performed, in addition to $O C T 4$ and $N A N O G$, as indicators of increased pluripotency and stemness. As expected, significantly higher $A C A N, C O L 2 A 1$ and SOX9 gene expression levels were identified in L-NPSCs in comparison with H-NPSCs. These results verified that the frequency of differentiated NP cells was increased in the cell population of L-NPSCs. Of note, the levels of OCT4 and NANOG, markers of multipotency, were found to be increased in L-NPSCs, which is in agreement with the study of Brisby et al (17). Taken together, these results suggest that the higher expression level of OCT4 and NANOG observed in L-NPSCs is due to an ongoing attempt to counter degenerative processes in the degenerated IVD, since L-NPSCs exhibited lower pluripotency.

Notably, the results of the present study demonstrated that H-NPSCs have a higher proliferative capacity and enhanced differentiation potential compared with L-NPSCs. The reduced proliferation capacity of L-NPSCs was in agreement with the results of cell cycle analysis indicating increased $\mathrm{G}_{0} / \mathrm{G}_{1}$ and decreased $\mathrm{G}_{2} / \mathrm{M}$ phase arrest and $\mathrm{S}$ phase entry. Furthermore, cell apoptosis analysis also confirmed this result by revealing that the percentage of necrotic and apoptotic cells among L-NPSCs was higher compared with H-NPSCs. The lower proliferation rate of L-NPSCs may be explained by the loss of positive MSC marker cells and the increase of CD24-positive cells. It is well-established that the proliferation capability of NP cells is weaker compared with that of MSC-like stem/progenitor cells (20). Additionally, a previous study also reported that the presence of CD24 was associated with inferior proliferation and with low colony-forming capability in NP tissues (30).

Multilineage potential was significantly higher in H-NPSCs compared with that in L-NPSCs with regard to adipogenic, chondrogenic and osteogenic differentiation potential. It was noted that, although the expression of the classic chondrogenic markers $S O X-9, C O L 2 A 1$ and $A C A N$ was markedly upregulated in L-NPSCs, L-NPSCs exhibited declined differentiation potential to chondrocytes, suggesting the L-NPSCs group contained a higher number of differentiated chondrocytes that bear little differentiation potential.

The results of the present study predominantly demonstrated that H-NPSCs with a higher intensity of MSC surface marker expression, and with an improved proliferative capacity and differentiation potential, were obtained from patients aged $<20$ years, while the majority of L-NPSC donors were aged $>25$ years, with one exception. Sakai et al (20) reported that the frequency of progenitor cells in NP tissues markedly decreased with age and degeneration of the IVD. Furthermore, Yasen et al (31) demonstrated that the number of endogenous progenitor cells and their proliferation capacity in rabbit IVD decreased with age. Additionally, vertebral mesenchymal stromal cells were demonstrated to decrease with age, which may be responsible for the age-associated osteoporosis (32). It has been hypothesized that the decline in the regenerative potential of NPSCs with age in IVD plays a key role in disc aging and degeneration (20). These findings offered novel insights into the biology of NPSCs during IVD degeneration. The limitations of the present study were that $\mathrm{H}-\mathrm{NPSC}$ were harvested from a relatively small sample size, and that H-NPSCs are infrequently obtained from degenerated NP tissues. However, the differences between L-NPSCs and $\mathrm{H}-\mathrm{NPSC}$ require further investigation and identification, and more studies on human NPSCs are required in order to provide an increased number of data sets.

In conclusion, the present study demonstrated that NPSCs with a higher expression intensity of MSC surface markers exhibited a higher proliferative capacity and differentiation potential, which may provide a novel strategy for evaluating the regenerative potential of NPSCs. Additionally, the present study may provide novel insights into the cellular mechanisms of IVD degeneration considering that NP resident stem cells serve a key role in maintaining the homeostasis of NP tissue.

\section{Acknowledgements}

Not applicable.

\section{Funding}

This study was supported by the Special Fund for Scientific Research Fostering of the First Affiliated Hospital, Jinan University, Guangzhou, Guangdong, China (grant no. 2017207), by the Science and Technology Program of Guangzhou, China (grant no. 201508020035, by the Special Fund of Science and Technology of Guangzhou Province (grant no.2017B030303001) and by the Science and Technology Program of Guangzhou, China (grant no. 201704020162).

\section{Availability of data and materials}

Supporting data and materials associated with this article can be accessed if required.

\section{Authors' contributions}

This study was designed by $\mathrm{JZ}$ and HW; data were collected by YS, JY, XZ and JL; data were analyzed and processed by JY, HW, MT and LHC; the manuscript was written by YS and JY, and revised by JZ. All the authors have read and approved the final version of the manuscript.

\section{Ethics approval and consent to participate}

All the procedures performed for the present study were approved by the medical ethics committee of Jinan University (Guangzhou, China). Specific informed consent was obtained in all cases.

\section{Patient consent for publication}

Not applicable.

\section{Competing interests}

All authors declare that there are no competing interests. 


\section{References}

1. Sakai D and Andersson GB: Stem cell therapy for intervertebral disc regeneration: Obstacles and solutions. Nat Rev Rheumatol 11: 243-256, 2015.

2. Breivik H, Eisenberg E and O'Brien T; OPENMinds: The individual and societal burden of chronic pain in Europe: The case for strategic prioritisation and action to improve knowledge and availability of appropriate care. BMC Public Health 13: 1229, 2013.

3. Millward-Sadler SJ, Costello PW, Freemont AJ and Hoyland JA: Regulation of catabolic gene expression in normal and degenerate human intervertebral disc cells: Implications for the pathogenesis of intervertebral disc degeneration. Arthritis Res Ther 11: R65, 2009.

4. Wang J, Markova D, Anderson DG, Zheng Z, Shapiro IM and Risbud MV: TNF- $\alpha$ and IL- $1 \beta$ promote a disintegrin-like and metalloprotease with thrombospondin type I Motif-5-mediated aggrecan degradation through syndecan-4 in intervertebral disc. J Biol Chem 286: 39738-39749, 2011.

5. Pattappa G, Li Z, Peroglio M, Wismer N, Alini M and Grad S: Diversity of intervertebral disc cells: Phenotype and function. J Anat 221: 480-496, 2012.

6. Zhao CQ, Wang LM, Jiang LS and Dai LY: The cell biology of intervertebral disc aging and degeneration. Ageing Res Rev 6: 247-261, 2007

7. Risbud MV, Albert TJ, Guttapalli A, Vresilovic EJ, Hillibrand AS, Vaccaro AR and Shapiro IM: Differentiation of mesenchymal stem cells towards a nucleus pulposus-like phenotype in vitro: Implications for cell-based transplantation therapy. Spine (Phila Pa 1976) 29: 2627-2632, 2004.

8. Weissman IL: Stem cells: Units of development, units of regeneration, and units in evolution. Cell 100: 157-168, 2000

9. Liu S, Liang H, Lee SM, Li Z, Zhang J and Fei Q: Isolation and identification of stem cells from degenerated human intervertebral discs and their migration characteristics. Acta Biochim Biophys Sin (Shanghai) 49: 101-109, 2017.

10. Henriksson HB, Svala E, Skioldebrand E, Lindahl A and Brisby H: Support of concept that migrating progenitor cells from stem cell niches contribute to normal regeneration of the adult mammal intervertebral disc: A descriptive study in the New Zealand white rabbit. Spine (Phila PA 1976) 37: 722-732, 2012.

11. Chuah YJ, Lee WC, Wong HK, Kang Y and Hee HT: Three-dimensional development of tensile pre-strained annulus fibrosus cells for tissue regeneration: An in-vitro study. Exp Cell Res 331: 176-182, 2015.

12. Gilbert HTJ, Hoyland, JA and Richardson, SM: Stem cell regeneration of degenerated intervertebral discs: Current status (Update). Curr Pain Headache Rep 17: 377, 2013.

13. Blanco JF, Graciani IF, Sanchez-Guijo FM, Muntión S, Hernandez-Campo P, Santamaria C, Carrancio S, Barbado MV, Cruz G, Gutierrez-Cosío S, et al: Isolation and characterization of mesenchymal stromal cells from human degenerated nucleus pulposus: Comparison with bone marrow mesenchymal stromal cells from the same subjects. Spine (Phila PA 1976) 35: 2259-2265, 2010.

14. Henriksson H, Thornemo M, Karlsson C, Hägg O, Junevik K, Lindahl A and Brisby $\mathrm{H}$ : Identification of cell proliferation zones, progenitor cells and a potential stem cell niche in the intervertebral disc region: A study in four species. Spine (Phila PA 1976) 34: 2278-2287, 2009.

15. Risbud MV, Guttapalli A, Tsai TT, Lee JY, Danielson KG, Vaccaro AR, Albert TJ, Gazit Z, Gazit D and Shapiro IM: Evidence for skeletal progenitor cells in the degenerate human intervertebral disc. Spine (Phila Pa 1976) 32: 2537-2544, 2007.

16. Mizrahi O, Sheyn D, Tawackoli W, Ben-David S, Su S, Li N, Oh A, Bae H, Gazit D and Gazit Z: Nucleus pulposus degeneration alters properties of resident progenitor cells. Spine J 13: 803-814, 2013.
17. Brisby H, Papadimitriou N, Brantsing C, Bergh P, Lindahl A, Barreto $\mathrm{H}$ and Henriksson: The presence of local mesenchymal progenitor cells in human degenerated intervertebral discs and possibilities to influence these in vitro: A descriptive study in humans. Stem Cells Dev 22: 804-814, 2013.

18. Wu H, Zeng X, Yu J, Shang Y, Tu M, Cheang LH and Zhang J: Comparison of nucleus pulposus stem/progenitor cells isolated from degenerated intervertebral discs with umbilical cord derived mesenchymal stem cells. Exp Cell Res 361: 324-332, 2017.

19. Wang W, Deng G, Qiu Y, Huang X, Xi Y, Yu J, Yang X and Ye X: Transplantation of allogenic nucleus pulposus cells attenuates intervertebral disc degeneration by inhibiting apoptosis and increasing migration. Int J Mol Med 41: 2553-2564, 2018.

20. Sakai D, Nakamura Y, Nakai T, Mishima T, Kato S, Grad S, Alini M, Risbud M, Chan D, Cheah K, et al: Exhaustion of nucleus pulposus progenitor cells with ageing and degeneration of the intervertebral disc. Nat Commun 3: 1264, 2012.

21. Conley BA, Koleva R, Smith JD, Kacer D, Zhang D, Bernabéu C and Vary CP: Endoglin controls cell migration and composition of focal adhesions: Function of the cytosolic domain. J Biol Chem 279: 27440-27449, 2004.

22. Molinos M, Almeida CR, Gonçalves RM and Barbosa MA: Improvement of bovine nucleus pulposus cells isolation leads to identification of three phenotypically distinct cell subpopulations. Tissue Eng Part A 21: 2216-2227, 2015.

23. Jia Z, Yang P, Wu Y, Tang Y, Zhao Y, Wu J, Wang D, He Q and Ruan D: Comparison of biological characteristics of nucleus pulposus mesenchymal stem cells derived from non-degenerative and degenerative human nucleus pulposus. Exp Ther Med 13: 3574-3580, 2017.

24. Turner S, Balain B, Caterson B, Morgan C and Roberts S: Viability, growth kinetics and stem cell markers of single and clustered cells in human intervertebral discs: Implications for regenerative therapies. Eur Spine J 23: 2462-2472, 2014.

25. Hegewald AA, Endres M, Abbushi A, Abraja CM, Woiciechowsky C, Schmieder K, Kaps C and Thomé C: Adequacy of herniated disc tissue as a cell source for nucleus pulposus regeneration. J Neurosurg Spine 14: 273-280, 2011.

26. Tekari A, Chan SC, Sakai D, Grad S and Gantenbein B: Angiopoietin-1 receptor Tie2 distinguishes multipotent differentiation capability in bovine coccygeal nucleus pulposus cells. Stem Cell Res Ther 23: 75, 2016.

27. Tang X, Jing L and Chen J: Changes in the molecular phenotype of nucleus pulposus cells with intervertebral disc aging. PLos One 7: e52020,2012.

28. Kim KW, Ha KY, Lee JS, Nam SW, Woo YK, Lim TH and An HS: Notochordal cells stimulate migration of cartilage end plate chondrocytes of the intervertebral disc in in vitro cell migration assays. Spine J 9: 323-329, 2009.

29. Sive JI, Baird P, Jeziorsk M, Watkins A, Hoyland JA and Freemont AJ: Expression of chondrocyte markers by cells of normal and degenerate intervertebral discs. Mol Pathol 55: 91-97, 2002.

30. Fujita N, Miyamoto T, Imai J, Hosogane N, Suzuki T, Yagi M, Morita K, Ninomiya K, Miyamoto K, Takaishi H, et al: CD24 is expressed specifically in the nucleus pulposus of intervertebral discs. Biochem Biophys Res Commun 338: 1890-1896, 2005.

31. Yasen M, Fei Q, Hutton WC, Zhang J, Dong J, Jiang X and Zhang F: Changes of number of cells expressing proliferation and progenitor cell markers with age in rabbit intervertebral discs. Acta Biochim Biophys Sin (Shanghai) 45: 368-376, 2013.

32. D'Ippolito G, Schiller PC, Ricordi C, Roos BA and Howard GA: Age-related osteogenic potential of mesenchymal stromal stem cells from human vertebral bone marrow. J Bone Miner Res 14: 1115-1122, 1999. 